\title{
Current-Voltage Characteristics in a Helium-Argon Gas Mixture Glow Discharge at Low Pressure
}

\author{
B. HeChelef And A. Bouchikhi*
}

University of Saïda, Faculty of Technology, Department of Electrical Engineering, Saïda 20000, Algeria

(Received March 24, 2018; revised version January 30, 2019; in final form October 2, 2019)

In this work, the current-voltage characteristics in a $90 \%$ helium-10\%argon gas mixture glow discharge at low pressure have been studied. Three pressures values are considered 1.5, 2, and 2.5 Torr. The range of voltage is between 150 and $500 \mathrm{~V}$. The model of our system is a fluid of two ordered components, in which the metastable state of the atoms and the radiation effects are included. The parameters of particle transport and their rate coefficients strictly depend on mean electron energy, and they are calculated by using the BOLSIG + software. The range of the mean electron energy is taken between 0.04 and $133 \mathrm{eV}$, and the electron energy distribution function is taken in non-Maxwellian form. The results show that the argon ion density is more important than the helium ion density, despite the presence of more constant background helium gas density in the mixture. The normal and abnormal glow discharge modes appear on the electric characteristics. The obtained results are compared to both experimental data and numerical results which exist in the literature.

DOI: 10.12693/APhysPolA.136.855

PACS/topics: gas discharge, Boltzmann's equation, Poisson's equation, Blanc's law, two order fluid model

\section{Introduction}

The technology of the glow discharge [1-7] is an important domain in the microelectronic industry such as the etching or the deposition of thin solid films, and a spectroscopic analytical for metallic and sputtering treatments. This technology is carried out a lot in pure gas, and in mixture gases. The current-voltage characteristics in the gas mixture were studied by several authors such as Kiselev et al. [8], who used nitrogen, carbon dioxide, and helium in the ratio 1:1:8, helium with carbon dioxide in the ratio $8: 1$, and also nitrogen with carbon dioxide in the ratio 1:1 flowing in narrow long tubes with various diameters. Although in a mixed gas, which includes only rare gas, is few in the literature. For this reason, in this paper, we propose a study in a helium-argon DC glow discharge at low pressure. By utilizing fluid model order two (containing the continuity and the momentum transfer equations and energy equation, and the set of these equations are coupled with Poisson's equation) we can find the electrical and energetic characteristics in each mixture gas, but the problem is related to the type and the percentage of gas in their mixture. To give best results by fluid model which are in good agreement to the experimental results, it is necessary to know several conditions, i.e., the electron energy distribution function (EEDF) is in a non-Maxwellian form and the percentage of the gas in the mixture is $90 \% \mathrm{He}-10 \% \mathrm{Ar}$.

In our previous research [9-11], we have found that the EEDF is non-Maxwellian for a threshold value of

\footnotetext{
* corresponding author; e-mail bouchikhiabdelaziz1@yahoo.fr
}

pressure, and again, it is related to the type of gas. The percentage of gas in the mixture is related to the ion mass. Note that the present study is carried out in a $1 \mathrm{~cm}$ inter-electrode spacing.

The objective of this paper is to determine the physical proprieties of a DC glow discharge at low pressure in $90 \% \mathrm{He}-10 \% \mathrm{Ar}$ in $1 \mathrm{~cm}$ inter-electrode spacing. In Sect. 2, kinetic scheme of processes and their modeling are detailed. Firstly, the different processes, which are intervening into the discharge mechanism are defined. Secondly, the mathematical model is described. Thirdly, the solution of the present model is discussed again. In Sect. 3, the results are analyzed. The conclusions are given in Sect. 4.

\section{Kinetic scheme of processes and their modeling}

In the helium-argon gas mixture DC glow discharge at low pressure, we can consider the chemical reaction processes and describe them separately with characteristic parameters. Determination of these parameters, known as the rate coefficients, is one of our purpose of our studies. The reactions for each gas component $\mathrm{X}$ in the mixture are the following:

- the elastic collision, i.e., $\mathrm{X}+\mathrm{e}^{-} \rightarrow \mathrm{X}+\mathrm{e}^{-}$

- ionization, i.e., $\mathrm{X}+\mathrm{e}^{-} \rightarrow \mathrm{X}^{+}+2 \mathrm{e}^{-}$

- excitation, $\mathrm{X}+\mathrm{e}^{-} \rightarrow \mathrm{X}_{m}^{*}+\mathrm{e}^{-}$

- de-excitation, $\mathrm{X}_{m}^{*}+\mathrm{e}^{-} \rightarrow \mathrm{X}+\mathrm{e}^{-}$

- chemoionization, $\mathrm{X}_{m}^{*}+\mathrm{X}_{m}^{*} \rightarrow \mathrm{X}^{+}+\mathrm{e}^{-}+\mathrm{X}$

- radiation process, $\mathrm{X}_{m}^{*} \rightarrow \mathrm{X}+h \nu$

- stepwise ionization, $\mathrm{e}^{-}+\mathrm{X}_{m}^{*} \rightarrow \mathrm{X}^{+}+2 \mathrm{e}^{-}$ 
The elastic collision processes are characterized by the rate coefficient $P_{\mathrm{ec}}^{\mathrm{gas}}(\mathrm{eV} / \mathrm{s})$. We compute them for argon and helium according to Refs. [12] and [13]. The ionization, excitation, and deexcitation processes are defined by the rate coefficients $K_{\text {io }}^{\text {gas }}\left(\mathrm{cm}^{3} / \mathrm{s}\right), K_{\mathrm{ex}}^{\text {gas }}\left(\mathrm{cm}^{3} / \mathrm{s}\right), \quad$ and $K_{\text {dex }}^{\text {gas }}\left(\mathrm{cm}^{3} / \mathrm{s}\right)$, respectively, and we compute them with BOLSIG $+[14,15]$. To determine the chemoionization rates $K_{\mathrm{ci}}^{\text {gas }}\left(\mathrm{cm}^{3} / \mathrm{s}\right)$ we follow approach in Ref. [16]. We obtain then $K_{\mathrm{ci}}^{\mathrm{Ar}}=5.2 \times 10^{-10} \mathrm{~cm}^{3} / \mathrm{s}$ and $K_{\mathrm{ci}}^{\mathrm{He}}=9.2 \times 10^{-10} \mathrm{~cm}^{3} / \mathrm{s}$. The radiation process in turn is well described by the rate coefficient $\tau_{m}^{\text {gas }}$. For helium gas we follow Ref. [17] and obtain $\tau_{m}^{\mathrm{He}}=1.7 \times 10^{-6} \mathrm{~s}$. In case of argon gas we follow Ref. [18] and obtain $\tau_{m}^{\mathrm{Ar}}=1 \times 10^{-7} \mathrm{~s}$. The stepwise ionization processes $K_{m \text { io }}^{\text {gas }}\left(\mathrm{cm}^{3} / \mathrm{s}\right)$ are calculated according to the approximation given by Vriens and Smeets [19].

By taking these chemical reactions, the fluid model in $90 \% \mathrm{He}-10 \% \mathrm{Ar}$ is written as follows:

$$
\begin{aligned}
& \frac{\partial n_{e}}{\partial t}+\frac{\partial \Gamma_{e}}{\partial x}=S_{e}, \\
& \frac{\partial n_{+}^{\mathrm{He}}}{\partial t}+\frac{\partial \Gamma_{+}^{\mathrm{He}}}{\partial x}=S_{+}^{\mathrm{He}}, \\
& \frac{\partial n_{+}^{\mathrm{Ar}}}{\partial t}+\frac{\partial \Gamma_{+}^{\mathrm{Ar}}}{\partial x}=S_{+}^{\mathrm{Ar}}, \\
& \frac{\partial n_{m}^{\mathrm{He}}}{\partial t}+\frac{\partial \Gamma_{m}^{\mathrm{He}}}{\partial x}=S_{m}^{\mathrm{He}}, \\
& \frac{\partial n_{m}^{\mathrm{Ar}}}{\partial t}+\frac{\partial \Gamma_{m}^{\mathrm{Ar}}}{\partial x}=S_{m}^{\mathrm{Ar}}, \\
& \frac{\partial \varepsilon_{e} n_{e}}{\partial t}+\frac{\partial \Gamma_{e \varepsilon}}{\partial x}=S_{e \varepsilon} .
\end{aligned}
$$

At low pressure, the destruction of the positive ions is negligible, and we can calculate the quantities $S_{m}^{\mathrm{He}}$ and $S_{m}^{\mathrm{Ar}}$ as follows [9-11, 20]:

$$
\begin{aligned}
& S_{+}^{\mathrm{He}}=n_{e}\left(0.9 n_{g} K_{\mathrm{io}}^{\mathrm{He}}+n_{m}^{\mathrm{He}} K_{m_{-}{ }_{\text {io }}}^{\mathrm{He}}\right)+n_{m}^{\mathrm{He}} n_{m}^{\mathrm{He}} K_{\mathrm{ci}}^{\mathrm{He}}, \\
& S_{+}^{\mathrm{Ar}}=n_{e}\left(0.1 n_{g} K_{\mathrm{io}}^{\mathrm{Ar}}+n_{m}^{\mathrm{Ar}} K_{m_{-} \text {io }}^{\mathrm{Ar}}\right)+n_{m}^{\mathrm{Ar}} n_{m}^{\mathrm{Ar}} K_{\mathrm{ci}}^{\mathrm{Ar}}, \\
& S_{e}=S_{+}^{\mathrm{He}}+S_{+}^{\mathrm{Ar}}, \\
& S_{m}^{\mathrm{He}}=n_{e}\left(0.9 n_{g} K_{\mathrm{ex}}^{\mathrm{He}}-n_{m}^{\mathrm{He}} K_{\mathrm{dex}}^{\mathrm{He}}-n_{m}^{\mathrm{He}} K_{m_{-} \text {iо }}^{\mathrm{He}}\right) \\
& -2 n_{m}^{\mathrm{He}} n_{m}^{\mathrm{He}} K_{\mathrm{ci}}^{\mathrm{He}}-\frac{n_{m}^{\mathrm{He}}}{\tau_{m}^{\mathrm{He}}}, \\
& S_{m}^{\mathrm{Ar}}=n_{e}\left(0.1 n_{g} K_{\mathrm{ex}}^{\mathrm{Ar}}-n_{m}^{\mathrm{Ar}} K_{\mathrm{dex}}^{\mathrm{Ar}}-n_{m}^{\mathrm{Ar}} K_{m_{-}{ }_{\text {io }}}^{\mathrm{Ar}}\right) \\
& -2 n_{m}^{\mathrm{Ar}} n_{m}^{\mathrm{Ar}} K_{\mathrm{ci}}^{\mathrm{Ar}}-\frac{n_{m}^{\mathrm{Ar}}}{\tau_{m}^{\mathrm{Ar}}}, \\
& \frac{\partial^{2} \varphi}{\partial x^{2}}=-\frac{e_{o}}{\varepsilon_{o}}\left(n_{+}^{\mathrm{He}}+n_{+}^{\mathrm{Ar}}-n_{e}\right),
\end{aligned}
$$

where $n_{e}, n_{+}^{\mathrm{He}}, n_{+}^{\mathrm{Ar}}, n_{m}^{\mathrm{He}}, n_{m}^{\mathrm{Ar}}$, and $\Gamma_{e}, \Gamma_{+}^{\mathrm{He}}, \Gamma_{+}^{\mathrm{Ar}}$, $\Gamma_{m}^{\mathrm{He}}, \Gamma_{m}^{\mathrm{Ar}}$ are particle densities, and particle flux of the electrons, helium, and argon ions, metastable atoms of helium and argon, respectively. $n_{g}$ denotes the neutral gas density. $S_{e}, S_{+}^{\mathrm{He}}, S_{+}^{\mathrm{Ar}}, S_{m}^{\mathrm{He}}$, and $S_{m}^{\mathrm{Ar}}$ represent source term of electrons, helium ion, argon ion, helium metastable atom, and argon metastable atom, respectively. $\varepsilon_{e}$ is the mean electron energy, $\Gamma_{e \varepsilon}$ is the electron energy flux. $S_{e \varepsilon}$ is the electron energy source term. $\varphi$ is the electrostatic potential, $E=-\partial \varphi / \partial x$ is the electric field strength. $\varepsilon_{o}$ and $e$ are the permittivity of free space and elementary charge, respectively.

The source term of energetic electrons represents the loss and product of electron energy, and it is computed from the multiplication of the source term of kinds of species and the corresponding energetic processes. As a result, we find the following expression:

$$
\begin{aligned}
& S_{e \varepsilon}=-e \Gamma_{e} E+\varepsilon_{m}^{\mathrm{Ar}} n_{e} n_{m}^{\mathrm{Ar}} K_{\mathrm{dex}}^{\mathrm{Ar}}+\varepsilon_{m}^{\mathrm{He}} n_{e} n_{m}^{\mathrm{He}} K_{\mathrm{dex}}^{\mathrm{He}} \\
& +\varepsilon_{\mathrm{ci}}^{\mathrm{Ar}} n_{m}^{\mathrm{Ar}} n_{m}^{\mathrm{Ar}} K_{\mathrm{ci}}^{\mathrm{Ar}}+\varepsilon_{\mathrm{ci}}^{\mathrm{He}} n_{m}^{\mathrm{He}} n_{m}^{\mathrm{He}} K_{\mathrm{ci}}^{\mathrm{He}}-n_{e} P_{\mathrm{ec}}^{\mathrm{Ar}} \\
& -n_{e} P_{\mathrm{ec}}^{\mathrm{He}}-n_{e}\left(\varepsilon_{m}^{\mathrm{Ar}} 0.1 n_{g} K_{\mathrm{ex}}^{\mathrm{Ar}}+\varepsilon_{\mathrm{io}}^{\mathrm{Ar}} 0.1 n_{g} K_{\mathrm{io}}^{\mathrm{Ar}}\right. \\
& +\left(\varepsilon_{\mathrm{io}}^{\mathrm{Ar}}-\varepsilon_{m}^{\mathrm{Ar}}\right) n_{m}^{\mathrm{Ar}} K_{m_{-} \text {io }}^{\mathrm{Ar}}+\left(\varepsilon_{\mathrm{io}}^{\mathrm{He}}-\varepsilon_{m}^{\mathrm{He}}\right) n_{m}^{\mathrm{He}} K_{m_{-} \text {io }}^{\mathrm{He}} \\
& \left.+\varepsilon_{m}^{\mathrm{He}} 0.9 n_{g} K_{\mathrm{ex}}^{\mathrm{He}}+\varepsilon_{\mathrm{io}}^{\mathrm{He}} 0.9 n_{g} K_{\mathrm{io}}^{\mathrm{He}}\right),
\end{aligned}
$$

where $\varepsilon_{m}^{\mathrm{He}}=19.8 \mathrm{eV}$ and $\varepsilon_{m}^{\mathrm{Ar}}=11.55 \mathrm{eV}$ are the corresponding energy of the excitation and de-excitation processes of helium and argon atoms, respectively. $\varepsilon_{\mathrm{io}}^{\mathrm{He}}=24.58 \mathrm{eV}$ and $\varepsilon_{\mathrm{io}}^{\mathrm{Ar}}=15.76 \mathrm{eV}$ are the energy losses by ionization processes of helium and argon atoms, respectively. The chemo-ionization processes relate to the energy gains by $\varepsilon_{\mathrm{ci}}^{\mathrm{He}}=2 \varepsilon_{m}^{\mathrm{He}}-\varepsilon_{\mathrm{io}}^{\mathrm{He}}$, and $\varepsilon_{\mathrm{ci}}^{\mathrm{Ar}}=2 \varepsilon_{m}^{\mathrm{Ar}}-\varepsilon_{\mathrm{io}}^{\mathrm{Ar}}$ of helium and argon atoms, respectively.

The particle flux of electrons, ions, metastable atoms, and electron energy are determined according to Becker et al. [20], Alili et al. [9] and they read

$$
\begin{aligned}
& \Gamma_{e}=-n_{e} \mu_{e} E-\frac{\partial D_{e} n_{e}}{\partial x}, \\
& \Gamma_{+}^{\mathrm{He}}=n_{+}^{\mathrm{He}} \mu_{+}^{\mathrm{He}} E-\frac{\partial D_{+}^{\mathrm{He}} n_{+}^{\mathrm{He}}}{\partial x}, \\
& \Gamma_{+}^{\mathrm{Ar}}=n_{+}^{\mathrm{Ar}} \mu_{+}^{\mathrm{Ar}} E-\frac{\partial D_{+}^{\mathrm{Ar}} n_{+}^{\mathrm{Ar}}}{\partial x}, \\
& \Gamma_{m}^{\mathrm{He}}=-D_{m}^{\mathrm{He}} \frac{\partial n_{m}^{\mathrm{He}}}{\partial x} \\
& \Gamma_{m}^{\mathrm{Ar}}=-D_{m}^{\mathrm{Ar}} \frac{\partial n_{m}^{\mathrm{Ar}}}{\partial x}, \\
& \Gamma_{e \varepsilon}=-n_{e} E \mu_{e \varepsilon}-\frac{\partial n_{e} D_{e \varepsilon}}{\partial x} .
\end{aligned}
$$

Here $\mu_{e}, \mu_{+}^{\mathrm{He}}, \mu_{+}^{\mathrm{Ar}}$, and $D_{e}, D_{+}^{\mathrm{He}}, D_{+}^{\mathrm{Ar}}$ denote the mobilities and diffusion coefficients of electron, helium, and argon ions in the mixture gas, respectively. The diffusion coefficient of metastable helium atoms is $D_{m}^{\mathrm{He}}=460 / p \mathrm{~cm}^{2} \mathrm{~s}^{-1}$ Torr, where $p$ is the gas pressure given in Torr [21]. The metastable argon atom diffusivity 
is $n_{g} D_{m}^{\mathrm{Ar}}=1.7 \times 10^{18} \mathrm{~cm}^{-1} \mathrm{~s}^{-1}[22]$. The helium ion mobility $\left(\mu_{+}^{\mathrm{He}}\right)$ in a helium-argon mixture is supposed to be equal to helium ion mobility in pure helium. Then, the drift velocity of a positive ion of helium in a pure helium gas [23] is given by

$$
w_{\mathrm{He}^{+}}^{\mathrm{He}}=\frac{29.6 E / n_{g}}{\left(1+0.01288 E / n_{g}\right)^{0.5}}
$$

The argon ion mobility $\left(\mu_{+}^{\mathrm{Ar}}\right)$ in a helium-argon mixture is calculated using Blanc's law [24]. Therefore, for the argon ion mobility in pure argon the drift velocity of positive ion of argon in pure argon [25] is

$$
w_{\mathrm{Ar}^{+}}^{\mathrm{Ar}}=\frac{\left(4 E / n_{g}\right)}{\left(1+\left(0.007 E / n_{g}\right)^{1.5}\right)^{0.33}} .
$$

The drift velocity of positive ion of argon in a pure helium gas is approximated by

$$
w_{\mathrm{Ar}^{+}}^{\mathrm{He}}=\frac{56 E / n_{g}}{\left(1+0.01239 E / n_{g}\right)^{0.4}},
$$

which is in good agreement with experimental results [26].

Einstein's relation [27] is used to determine the helium ion diffusivity in a helium-argon mixture $\left(D_{+}^{\mathrm{He}}\right)$ as well as the argon ion diffusivity $\left(D_{+}^{\mathrm{Ar}}\right)$ in a heliumargon gas mixture. $D_{e \varepsilon}$ and $\mu_{e \varepsilon}$ represent the diffusion coefficient and mobility of electron energy, respectively and $D_{e \varepsilon}=D_{e} \varepsilon_{e} 5 / 3 ; \mu_{e \varepsilon}=\mu_{e} \varepsilon_{e} 5 / 3$. The parameter transports of electrons in $9 \% \mathrm{He}-10 \% \mathrm{Ar}$ strictly depend on mean electron energy, and are calculated by BOLSIG+ software [14, 15].

\subsection{Initial and boundary conditions}

Figure 1 represents the geometry of the reactor of plasma, which is used in our study. The initial and boundary conditions employed in $\mathrm{He}-\mathrm{Ar}$ gas mixture are detailed below.

Initial densities of electron, argon ion, and helium ion as well as argon and helium metastable atoms and mean electron energy are given as follows:

$$
\left\{\begin{array}{l}
n_{e}=n_{m}^{\mathrm{He}}=n_{m}^{\mathrm{Ar}}=10^{3} \mathrm{~cm}^{-3}, \\
n_{+}^{\mathrm{He}}=n_{+}^{\mathrm{Ar}}=0.5 \times 10^{3} \mathrm{~cm}^{-3}, \\
\varepsilon_{e}=1 \mathrm{eV} .
\end{array}\right.
$$

The boundary conditions at the cathode are

$$
\left\{\begin{array}{l}
\Gamma_{e}=-\gamma_{\mathrm{He}} \Gamma_{+}^{\mathrm{He}}-\gamma_{\mathrm{Ar}} \Gamma_{+}^{\mathrm{Ar}}, \\
n_{m}^{\mathrm{He}}=n_{m}^{\mathrm{Ar}}=0, \\
\varepsilon_{e}=5 \mathrm{eV}
\end{array}\right.
$$

where $\gamma_{g a s}$ represents the secondary electron emission coefficient corresponding to each gas. The bombardment of the positive ions of each gas (helium and argon) under the electric field will extract electron number from material cathode, where this probability of the release is represented by $\gamma_{\text {gas }}$.

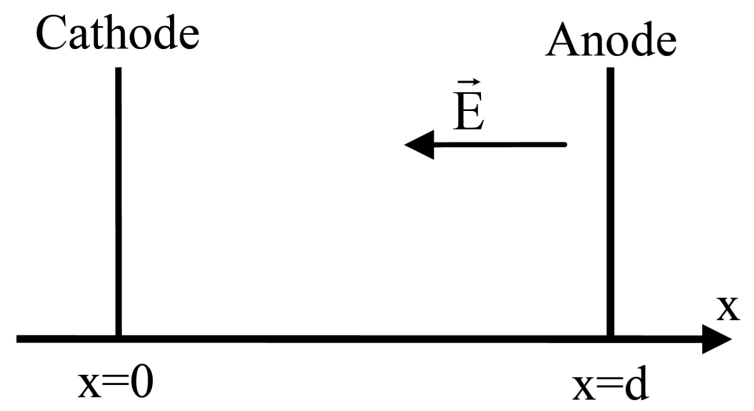

Fig. 1. Electrodes geometry for $\mathrm{He}-\mathrm{Ar}$ gas mixture glow discharges.

The boundary conditions at the anode are

$$
\left\{\begin{array}{l}
\frac{\partial n_{+}^{\mathrm{He}}}{\partial x}=0, \\
\frac{\partial n_{+}^{\mathrm{Ar}}}{\partial x}=0, \\
n_{m}^{\mathrm{He}}=n_{m}^{\mathrm{Ar}}=n_{e}=0 .
\end{array}\right.
$$

The Neumann boundary condition of the ion densities is used for the numerical reasons, with which our calculation is carried out from 2 to $N_{x}-1\left(N_{x}\right.$ represents the maximum number of the grid discretization), and this condition has no influence on the physical discharge. The discretizations of the differential equations (Eqs. (1)-(19)) are carried out by Scharfetter and Gummel method [28].

\section{Results and discussion}

The results show that the argon ion density is more important than the helium ion density despite the presence of more constant background helium gas density in the mixture. This observation is right for all gas pressures considered and all applying voltages. For example, Fig. 2 shows the spatial distributions of the electron, helium ion, and argon ion densities in a $90 \% \mathrm{He}-10 \% \mathrm{Ar}$ gas mixture in the stationary state of the discharge at $p=1.5$ Torr, $V_{\mathrm{DC}}=250 \mathrm{~V}$ and at $p=2.5$ Torr, $V_{\mathrm{DC}}=325 \mathrm{~V}$. This figure confirms our ascertainment, i.e., the argon ion density is more significant compared to the helium ion density (see Fig. 2).

Figure 3 represents the current-voltage characteristics in $90 \% \mathrm{He}-10 \% \mathrm{Ar}$ gas mixture glow discharge at low pressure. Note that the current density values are processed by "log" function. The inter-electrode spacing is taken to be constant and it is equal to $1 \mathrm{~cm}$. The gas temperature is equal to $300 \mathrm{~K}$. The secondary electron emission coefficient is supposed to be constant, and it is equal to 0.3 for positive helium ions [29] and it is equal to 0.06 for positive argon ions [25]. Note that all points in Fig. 3 are taken from the spatial distributions of the current densities, which are strictly constants in all space in inter-electrode, i.e., for the variable spatial distributions of current densities not being considered. Note also that these distributions are taken in the stationary 

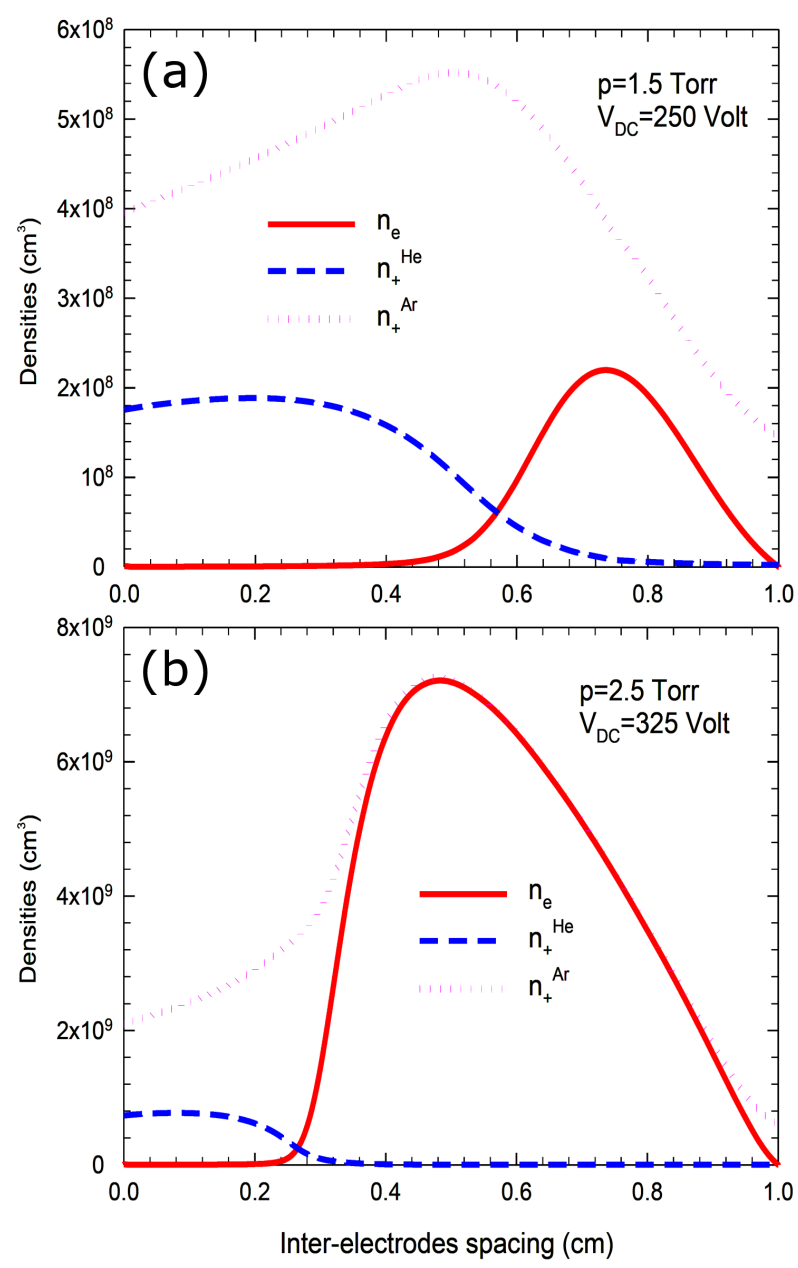

Fig. 2. Spatial distributions of the electron, helium ion and argon ion densities in a $90 \% \mathrm{He}-10 \% \mathrm{Ar}$ gas mixture in the stationary state, (a) at 1.5 Torr and $V_{\mathrm{DC}}=250 \mathrm{~V}$, (b) at 2.5 Torr and $V_{\mathrm{DC}}=325 \mathrm{~V}$.

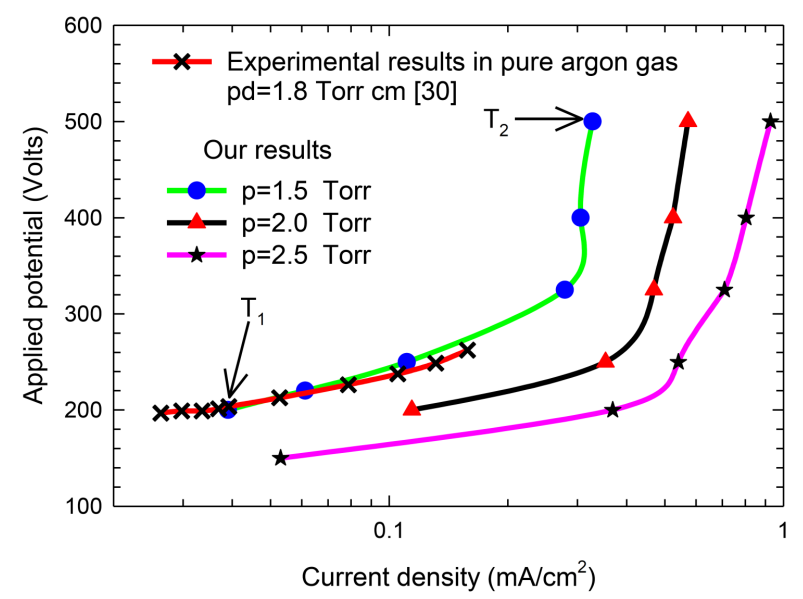

Fig. 3. Current-voltage characteristics in $90 \% \mathrm{He}^{-}$ $10 \%$ Ar gas mixture. With $T_{1}=7 \times 10^{-5} \mathrm{~s}$, and $T_{2}=2.5 \times 10^{-5} \mathrm{~s}$, which represent the maximum time of simulation, and it again represents the study state of the discharge. state of the discharge. The stationary state of the discharge depends of the gas pressure and applying potential of the electrodes. For example, the point (1.5 Torr, $200 \mathrm{~V})$ converges at $T_{1}=7 \times 10^{-5} \mathrm{~s}$, and the point (1.5 Torr, $500 \mathrm{~V}$ ) converges at $T_{2}=2.5 \times 10^{-5} \mathrm{~s}$. We remark that the applied potential plays an important role to accelerate the discharge convergence. In order to examine these curves, i.e., the current-voltage characteristic at different pressures (see Fig. 3), we observe that these curves are characterized by two modes, i.e., the normal and abnormal glow discharge modes. For example, the abnormal glow discharge mode is defined in the range (superior of $300 \mathrm{~V}$ ) at 1.5 Torr. The same curves have been observed by several experimental studies in pure gas. Note that the spatial distribution of the argon ion density is important to the helium ion density despite the presence of important percentage of the helium in the mixture. This is due to the value of threshold ionization, which is smaller for argon $\left(\varepsilon_{\mathrm{io}}^{\mathrm{Ar}}=15.76 \mathrm{eV}\right)$ than for helium $\left(\varepsilon_{\mathrm{io}}^{\mathrm{He}}=24.58 \mathrm{eV}\right)$. Therefore, the production of argon ions is more efficient than of helium ions during the ionization process. This phenomenon is also observed in the case of $\mathrm{Ne}-\mathrm{Xe}$ gas mixture [10], and $\mathrm{Ne}-\mathrm{Ar}$ gas mixture [11]. To conclude, the current-voltage characteristics in $90 \% \mathrm{He}-10 \% \mathrm{Ar}$ gas mixture approach the current-voltage characteristics in pure argon gas for a certain range of the electric potential, which are shown in Fig. 3 comparing experimental results obtained in pure argon gas [30].

Figure 4 represents the maximum values of the helium and argon metastable atom densities as a function of electric potential in a $90 \% \mathrm{He}-10 \% \mathrm{Ar}$ gas mixture at three pressures 1.5, 2, and 2.5 Torr. We note that the helium metastable atom density is superior to the argon metastable atom density because of the presence of an important percentage of the helium in the mixture.

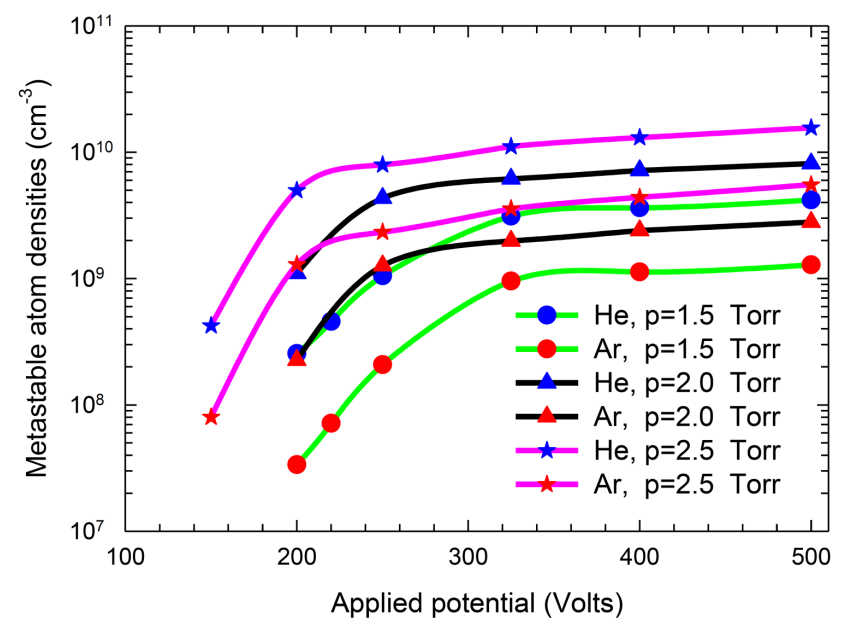

Fig. 4. The maximum values of the helium and argon metastable atom densities as a function of electric potential in a $90 \% \mathrm{He}-10 \% \mathrm{Ar}$ gas mixture at the pressures 1.5, 2, and 2.5 Torr. 


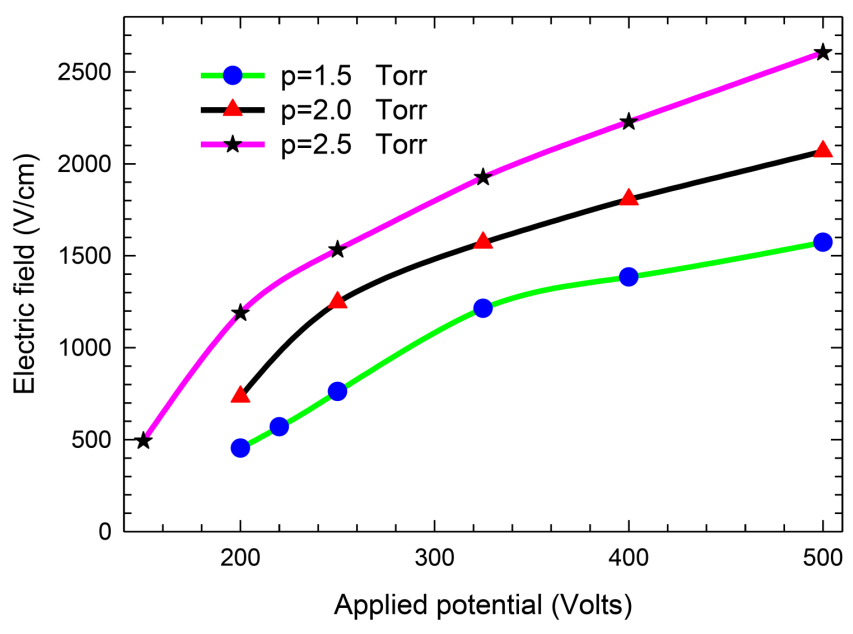

Fig. 5. Electric field at the cathode as a function of electric potential in a $90 \% \mathrm{He}-10 \% \mathrm{Ar}$ gas mixture at the pressure $1.5,2$, and 2.5 Torr.

We remark that the pressure and applying voltage have strong consequences on the metastable atom densities for each gas in the mixture, where the metastable atom densities increase with pressure and the electric potential, too. These are due to the decrease of the free path and augmentation of the electric field, which causes a lot of excitation collisions, respectively.

Figure 5 represents the electric field at the cathode as a function of electric potential in a $90 \% \mathrm{He}-$ $10 \%$ Ar gas mixture at the pressures 1.5, 2, and 2.5 Torr. We observe that the electric field increases with augmentation of the electric potential, which is absolutely evident when the formulation of the gradient of the electric potential is utilized. Note that the electric field increases with pressure due to the augmentation of the particle densities.

Figure 6 represents the maximum values of the mean electron energy as a function of electric potential in a $90 \% \mathrm{He}-10 \% \mathrm{Ar}$ gas mixture at the pressure $1.5,2$, and 2.5 Torr. We note that the maximum value recorded in this study is approximately $100 \mathrm{eV}$. This strong value is due to the effect of the helium gas in the mixture. In view of this figure, we conclude an important remark, which is that the mean electron energy decreases with increase of the pressure for an range voltage, and the mean electron energy increases with increase in the pressure for another range voltage. As example for the range $(>300 \mathrm{~V})$, we remark that the mean electron energy at the pressure 1.5 Torr is slightly superior to the mean electron energy at the pressure 2.5 Torr. For the range $(<300 \mathrm{~V})$, the mean electron energy at the pressure 2.5 Torr is superior to the mean electron energy at the pressure 1.5 Torr. These observations are related to the type of the glow discharge mode, i.e., in the normal glow discharge mode, the mean electron energy increases with pressure, and in the abnormal glow discharge mode the mean electron energy decreases with augmentation of the gas pressure.

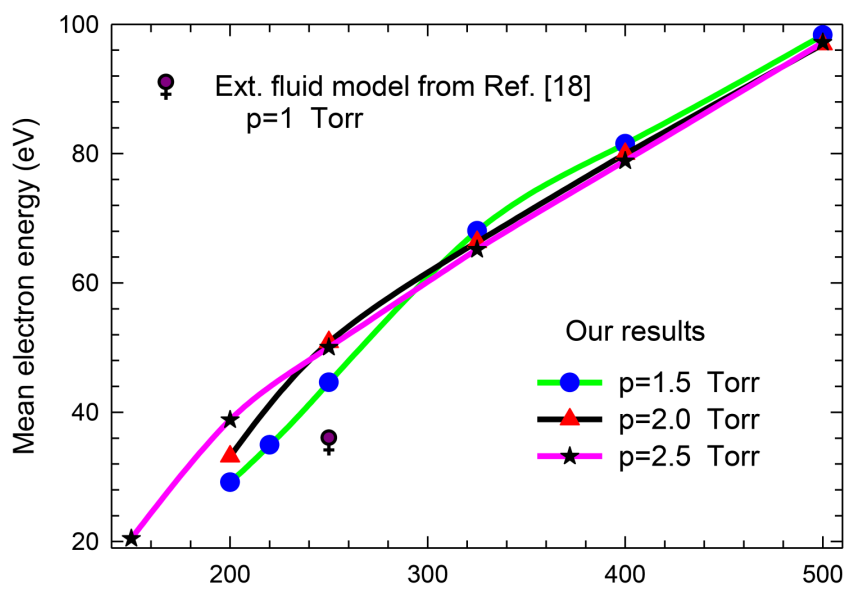

Fig. 6. The maximum values of the mean electron energy as a function of the electric potential in a $90 \% \mathrm{He}-$ $10 \%$ Ar gas mixture at the pressures $1.5,2$ and 2.5 Torr.

These results are compared to the results obtained by extended fluid model in pure argon gas [18] with $p=1$ Torr. We note that the mean electron energy increases with pressure, but this increase stops at 2 Torr due to the decrease of the mean free path.

\section{Conclusion}

The fluid model has been used to calculate the currentvoltage characteristics in a $\mathrm{He}-\mathrm{Ar}$ gas mixture glow discharge at low pressure. The Scharfetter and Gummel scheme has been used to discretize differential equations of the transport particles, and Thomas's scheme has been used for resolution of each equation. The transport parameters and the rate coefficients strictly depend on mean electron energy, which leads to make the situation of the discharge very correct and the results are close to the experimental results. The normal and abnormal glow discharge modes have appeared in the current-voltage characteristics. These results present good reference in the literature.

\section{References}

[1] B. Chapman, Glow Discharge Processes, Wiley, New York 1980

[2] W.W. Harrison, Glow Discharge Mass Spectrometry, Wiley, New York 1988.

[3] R.K. Marcus, Glow Discharge Spectroscopies, Plenum Press, New York 1993.

[4] M. Meyyappan, J.P.L. Kreskovsky, J. Appl. Phys. 68, 1506 (1990).

[5] A. Bogarets, R. Gijbels, W.J. Goedheer, J. Appl. Phys. 78, 2233 (1995).

[6] A. Bouchikhi, A. Hamid, Plasma Sci. Technol. 12, 59 (2010).

[7] B. Hechelef, A. Bouchikhi, Plasma Sci. Technol. 20, 115401 (2018). 
[8] A.S. Kiselev, E.A. Smirnov, A.A. Zagoskin, IOP Conf. Ser. Mater. Sci. Eng. 387, 012035 (2018).

[9] T. Alili, A. Bouchikhi, M. Rizouga, Can. J. Phys. 94, 731 (2016).

[10] A. Bouchikhi, Plasma Sci. Technol. 19, 095403 (2017).

[11] A. Bouchikhi, Can. J. Phys. 96, 62 (2018).

[12] F. Sigeneger, R. Winkler, IEEE Trans. Plasma Sci. 27, 1254 (1999).

[13] W. Van Gaens, A. Bogaerts, J. Phys. D Appl. Phys. 47, 079502 (2014).

[14] G.J.M. Hagelaar, L.C. Pitchford, Plasma Sources Sci. Tech. 14, 722 (2005).

[15] Plasma Data Exchange Project.

[16] N.B. Kolokolov, A.A. Kudrjavtsev, A.B. Blagoev, Phys. Scr. 50, 371 (1994).

[17] P. Maximilien, Ph.D. Thesis, Université Paris VI, 2007.

[18] I. Rafatov, E.A. Bogdanov, A.A. Kudryavtsev, Phys. Plasma 19, 093503 (2012).

[19] L. Vriens, A.H.M. Smeets, Phys. Rev. A 22, 940 (1980).
[20] M.M. Becker, D. Loffhagen, W. Schmidt, Comp. Phys. Com. 180, 1230 (2009).

[21] L.A. Palkina, B.M. Smirnov, M.I. Chibisov, Sov. Phys. JETP 29, 187 (1969).

[22] A.V. Phelps, J.P. Molnar, Phys. Rev. 89, 1202 (1953).

[23] L.S. Frost, Phys. Rev. 105, 354 (1957).

[24] A. Blanc, J. Phys. 7, 825 (1908).

[25] A.V. Phelps, Z.Lj. Petrovic, Plasma Sources Sci. Techn. 8, R21 (1999).

[26] H.W. Ellis, R.Y. Pai, E.W. Mcdaniel, E.A. Mason, L.A. Viehland, At. Data Nucl. Data Tables 17, 177 (1976).

[27] A. Bouchikhi, Plasma Sci. Technol. 14, 965 (2012).

[28] D.L. Scharfetter, H.K. Gummel, IEEE Trans. Electron. Dev. 16, 64 (1969).

[29] Z. Donkó, Phys. Rev. E 57, 7126 (1998).

[30] V.Lj. Marković, S.R. Gocić, S.N. Stamenković, Phys. Plasmas 12, 073502 (2005). 